\title{
Gesamtzustand des Patienten sollte vor der OP berücksichtigt werden
}

\begin{abstract}
Die bisherigen Kriterien für den Transplantatempfänger beziehen sich vorrangig auf die organbezogenen funktionellen Einschränkungen, also die Schwere der Lungenerkrankung. Ein wesentlicher Prädiktor für das Ergebnis einer Lungentransplantation ist aber auch der Gesamtzustand des Patienten. Die Bestimmung der Musculus-psoas-Querschnittsfläche im CT ist dabei ein gutes Maß für die Gebrechlichkeit.
\end{abstract}

Die Lungentransplantation ist für viele Patienten mit einer fortgeschrittenen Lungenerkrankung wie Mukoviszidose, COPD und interstitielle Lungenerkrankungen die Ultima Ratio. Nach den jährlich veröffentlichten Daten der International Society for Heart and Lung Transplantation nimmt der Anteil der Patienten mit interstitiellen Lungenerkrankungen bzw. Fibrosen, die einer Transplantation zugeführt werden, zu, während die Zahlen für COPD etwa gleich bleiben.

Das mediane Überleben nach Lungentransplantation beträgt heute 5,7 Jahre, die 1-Jahres-Überlebensrate $80 \%$. Nach 5 Jahren leben noch $54 \%$ und nach 10 Jahren noch $31 \%$ der transplantierten Patienten. Die häufigste Todesursache nach der Transplantation ist das frühe Transplantatversagen mit $24,3 \%$ gefolgt von Infektionen mit 19,3\%. Ein erhöhtes Risiko für frühes Transplantatversagen haben Patienten mit interstitiellen Lungenerkrankungen bzw. idiopathischer pulmonal arterieller Hypertonie und mit Blutgruppeninkompatibilität, Beatmung oder Dialyse. [1].

\section{Bewertung der Frailty ist sinnvoll}

Bisher sind die entscheidenden Kriterien bei der Indikationsstellung für die Lungentransplantation die spezifisch organbezogenen funktionellen Einschränkungen. „Der Gesamtzustand bleibt abgesehen von groben Einschätzungen mittels BodyMass-Index unberücksichtigt“", erläuterte Prof. Jürgen Behr, Asklepios Fachkliniken München-Gauting. Doch ein wichtiger Prädiktor für den Erfolg einer Lungentransplantation sei auch der Allgemeinzustand, genauer gesagt die Gebrechlichkeit. Eine quantitative Bewertung der Gebrechlichkeit ist allerdings schwierig und die Ergebnisse werden auch stark vom Untersuchungsinstrument bzw. dem Assessment beeinflusst.

In einer neuen Analyse wurde jetzt gezeigt, dass die mittels CT bestimmte Querschnittsfläche des Musculus psoas eine sinnvolle und zuverlässige Methode ist ( $\wedge A b b .1)$, um die körperliche Verfassung eines Patienten und damit den möglichen Erfolg einer Transplantation besser beurteilen zu können [2]. „Dieser Parameter korreliert negativ mit der Dauer der mechanischen Beatmung, der Notwendigkeit einer Tracheotomie, der Dauer des Aufenthaltes auf Intensivstation und der 6-Minutengehstrecke nach Rehabilitation“, erläuterte Behr. Dieses Kriterium sollte deshalb zumindest bei Patienten mit grenzwertiger Indikation als zusätzlicher Parameter herangezogen werden.
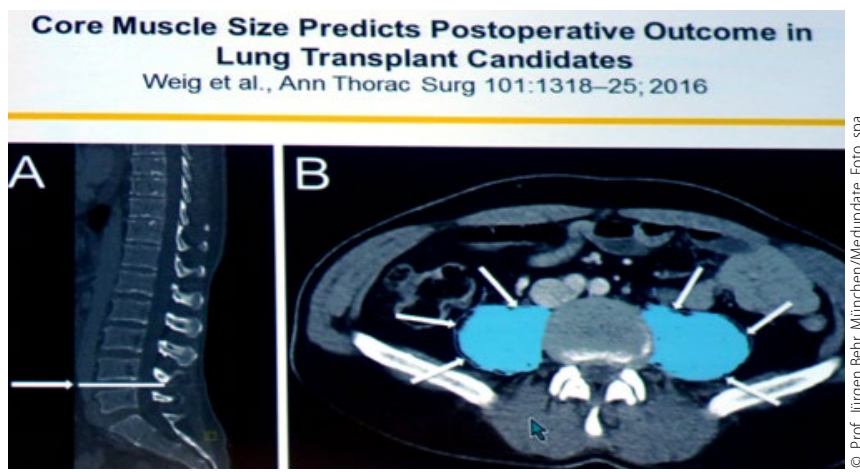

1 Über die Bestimmung der Querschnittsfläche des M. psoas in der CT kann die Verfassung eines Patienten zuverlässig beurteilt werden.

Die Frage, ob eine einseitige oder eine beidseitige Lungentransplantation die bessere Option ist, wird seit vielen Jahren kontrovers diskutiert. Ein wichtiges Argument für die einseitige Transplantation ist es, dass man dann mit einem Spenderorgan zwei Patienten helfen kann. Registerdaten zeigen, dass die doppelseitige Transplantation im Vergleich zur einseitigen bei Patienten mit einer idiopathischen Lungenfibrose mit einem signifikanten Überlebensvorteil einhergeht, bei COPD-Patienten ergab sich nach fünf Jahren allerdings kein Unterschied [3].

\section{Chronisches Transplantatversagen: FEV $_{1}+$ FVC messen} Die Prüfung der Organfunktion nach einer Lungentransplantation erfolgt meistens mittels Spirometrie, wobei die Bestimmung des $\mathrm{FEV}_{1}$ vorrangig ist. „Ein Abfall von $\mathrm{FEV}_{1}$ ist der entscheidende Hinweis für ein Organversagen", so Behr. Aber auch die forcierte Vitalkapazität (FVC) ist prognostisch relevant. Die beste Aussagekraft hat daher die Bestimmung beider Parameter. Wenn $\mathrm{FEV}_{1}$ und FVC jeweils um $\geq 20 \%$ fallen, so ist dies ein sehr zuverlässiges Kriterium für einen schlechten Verlauf [4]. Um Prognose und postoperativen Verlauf besser beurteilen zu können, sollte darum immer beides bestimmt werden.

Beim chronischen Lungenversagen werden unterschiedliche Typen unterschieden. Prognostisch ungünstiger ist der radiomorphologische Phänotyp des Restrictiv Allograft Syndroms (RAS). „Es bleibt jedoch offen, ob ein Abfall beider Parameter eher für ein RAS spricht", so Behr.

Dr. med. Peter Stiefelhagen

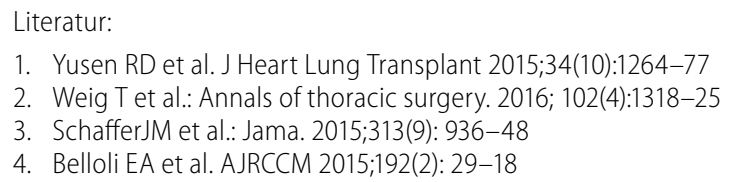

Quelle: Pneumo Update, 18. November 2016 in Wiesbaden 